\title{
Combination of a vascular plug and coiling to treat an ascending aortic pseudloaneurysm following aortic surgery
}

\begin{abstract}
Yuta Kikuchi, MD, Shingo Kunioka, MD, Tomonori Shirasaka, MD, PhD, and Hiroyuki Kamiya, MD, PhD, Asahikawa, Japan
\end{abstract}

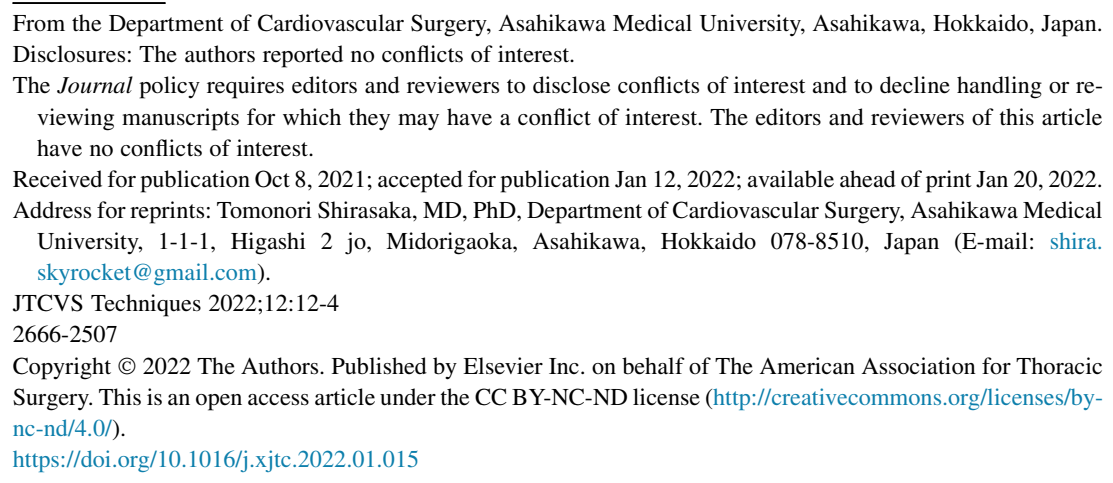

$\checkmark$ Video clip is available online.

Ascending aortic pseudoaneurysm (AAP) is a rare disease occurring in patients with aortic trauma, infectious aortitis, or after cardiovascular surgery. ${ }^{1}$ Sullivan and colleagues ${ }^{2}$ reported the potential sites of AAPs. AAPs are often located posterior to the sternum, and patients with AAPs have other comorbidities. Therefore, sternotomy (or resternotomy) is a risk factor for rupture.

We report a case in which an Amplatzer vascular plug II (AVPII; Abbott) and coiling were combined to treat an AAP. This technique can be used in high-risk cases, such as patients who are very frail and those with relatively large AAPs.

\section{CASE PRESENTATION}

A 71-year-old man who underwent total arch replacement with frozen elephant trunk to ensure closer distal anastomosis and coronary artery bypass grafting 4 years previously (Figure 1, A) was diagnosed with an AAP. The origin of the aneurysm was the proximal anastomotic site where coronary artery bypass grafting was performed, and it was located posterior to the sternum. The AAP measured $37 \times 46 \times 50 \mathrm{~mm}$, and the size of the entry point was approximately $9 \mathrm{~mm}$ (Figure $1, B-D$ ). Considering the position of the AAP and the risk of surgery (European System for Cardiac Operative Risk Evaluation II was $21.71 \%$ if thoracic surgery was opted), endovascular treatment (EVT) combined with AVPII

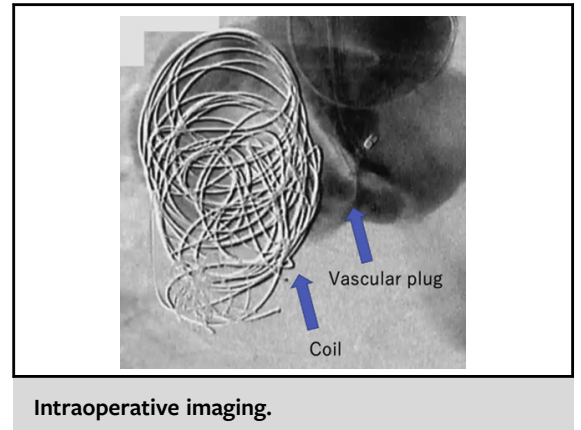

\begin{abstract}
CENTRAL MESSAGE
A combination of AVP and coil-

ing is an effective technique for

the treatment of AAP that

cannot be treated with a stent graft.
\end{abstract}

See Commentary on page 15.

and coiling was planned. We obtained the patient's consent for the off-label use of these devices. The patient also had a residual thoracic aortic aneurysm in the thoracic descending aorta. Therefore, we decided to perform thoracic endovascular aortic repair (TEVAR) simultaneously.

TEVAR was performed via the right femoral artery followed by insertion of a 6-Fr parent sheath (Parent Plus 60; Medikit) into the right brachial artery for AVPII and coiling. Aortography underwent to detect the entry site of the AAP. An Interlock-35 coil $(20 \times 400$ mm; Boston Scientific) was placed in the AAP to facilitate thrombosis, and an AVPII (9AVP2-016; Abbott) was placed at the entry site. Afterwards, we placed additional coils $(24 \times 600 \mathrm{~mm}, 28 \times 600 \mathrm{~mm} \times 3$ pieces, Ruby coil; Penumbra) in the AAP. Residual blood flow into the aneurysm was observed, but we finished the procedure and expected thrombosis to occur. The patient's postoperative course was uneventful, and the patient was transferred to another rehabilitation hospital with antiplatelet therapy for coronary artery disease at 1 month postoperatively. Six months postoperatively, an enhanced computed tomography scan 

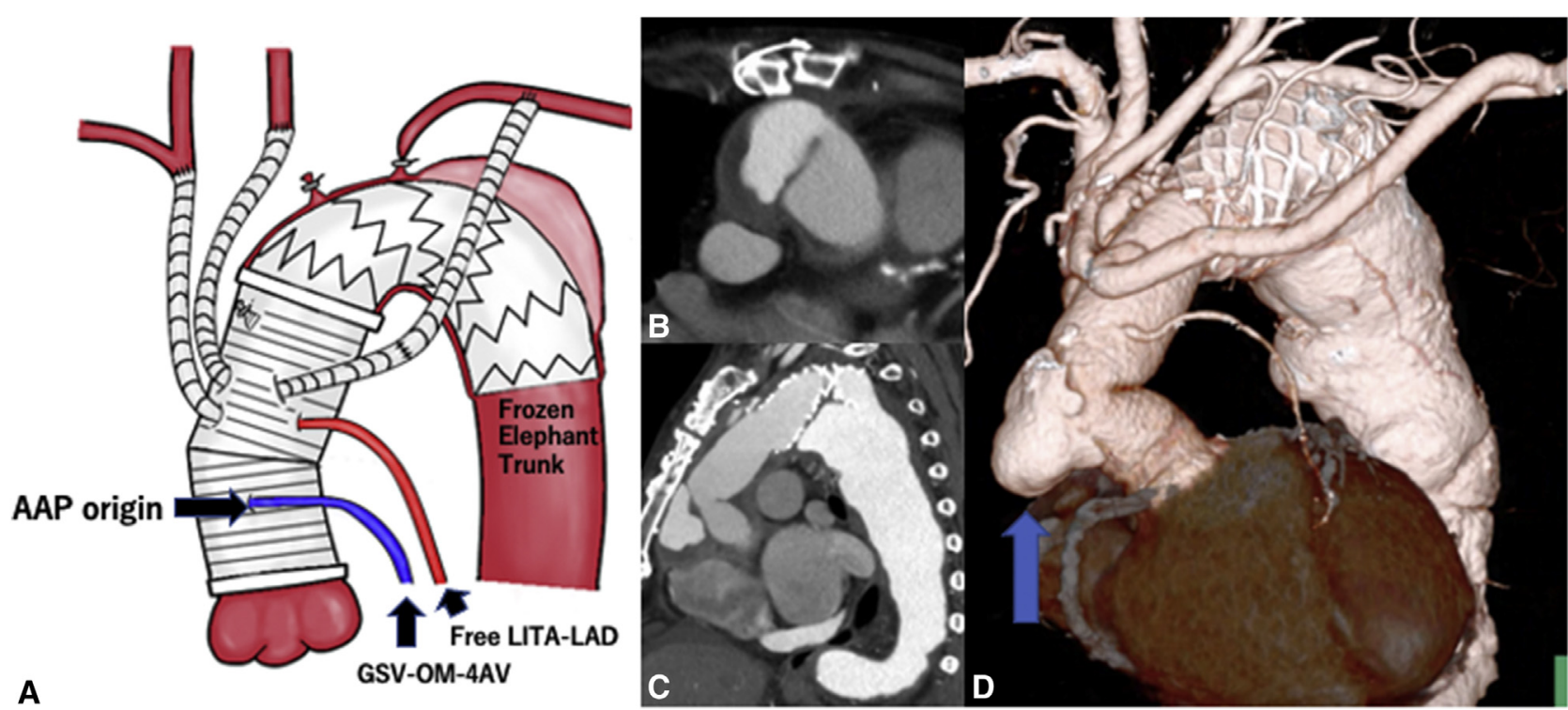

FIGURE 1. Preoperative enhanced computed tomography. A, Scheme of the previous surgery, B, Axial view; C, sagittal view; and D, 3-dimensional (3D)CT. Blue arrow shows ascending aortic pseudoaneurysm (AAP). LITA-LAD, Left internal thoracic artery to left anterior descending; GSV-OM-4AV, Great saphenous vein to obtuse marginal branch and atrioventricular node branch.

taken at the rehabilitation hospital revealed that the size of the AAP had decreased significantly (Figure 2 and Video 1). We plan to perform enhanced computed tomography every 6 months to follow-up the AAP size.

\section{DISCUSSION}

AAPs can occur after cardiac surgeries. ${ }^{1}$ The operative strategy for the treatment of AAP is complicated and is determined by its position, size, and site of entry.

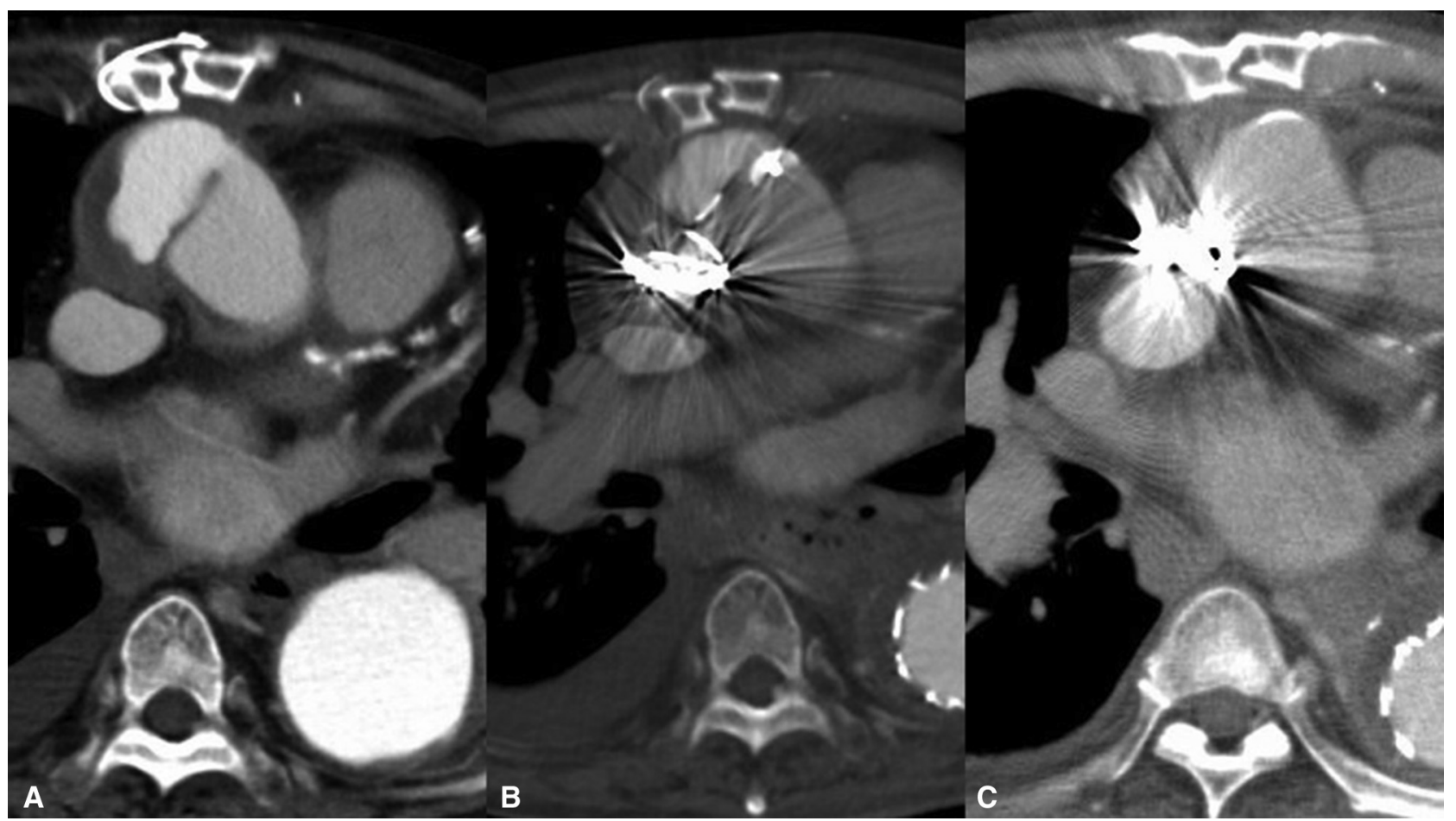

FIGURE 2. Enhanced computed tomography showing time course of ascending aortic pseudoaneurysm shrinkage. A, Preoperative; B, at 1 week postoperatively (Video 2); C, at 6 months' postoperatively. 


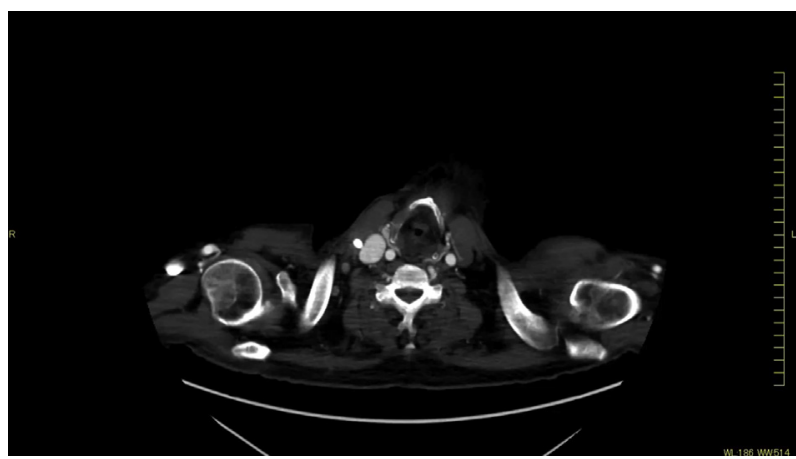

VIDEO 1. Time course of the patient with preoperative computed tomography (CT) scan, intraoperative image, and enhanced CT scan at 6 months postoperatively. Video available at: https://www.jtcvs.org/article/S26662507(22)00045-1/fulltext.

Resternotomy is associated with the risk of aneurysmal rupture; therefore, EVT is sometimes considered and there have been some reports on EVT using TEVAR, vascular plug, or coils..$^{3-5}$

There has been a report of TEVAR for ascending aortic disease, ${ }^{3}$ and we consider it the best option to occlude the entry site. However, we require sufficient length for the landing zone of TEVAR. De Boo and colleagues ${ }^{4}$ reported a case in which a combined technique comprising AVPII and coiling was used, similar to our case. This technique is useful for the treatment of a relatively large AAP that cannot be managed using TEVAR.

To occlude the entry site, some studies reported the off-label use of atrial septal defect or ventricular septal defect occluders, ${ }^{4}$ which are useful in closing AAPs with large entry sites owing to the structure. However, the case is limited by the AAP anatomy and an experienced physician is mandated owing to the certification and difficulty.

Considering the aforementioned discussion, we suggest that cases that can be treated with TEVAR should be managed using TEVAR. In addition, cases that cannot be treated with TEVAR should be treated using a combination of AVP and coiling to facilitate thrombosis in the AAP. In the applicable cases, to prevent migration, atrial septal defect or ventricular septal defect occluders are considered better than AVP or AVPII.

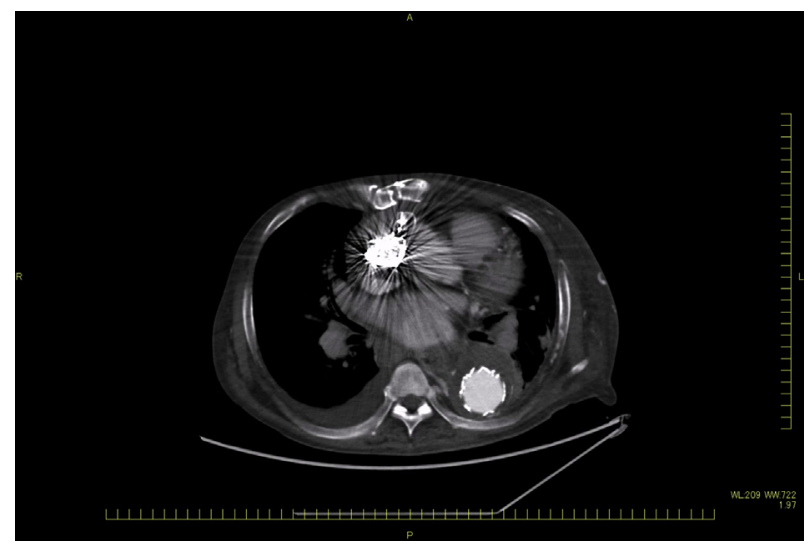

VIDEO 2. Enhanced computed tomography (CT) at 1 week postoperatively. Video available at: https://www.jtcvs.org/article/S2666-2507(22) 00045-1/fulltext.

\section{CONCLUSIONS}

Herein, we report a case of AAP that was treated using a combined technique comprising the use of AVPII and coiling. This technique is a good option for the treatment of AAP that cannot be treated by open surgery or TEVAR. However, there are no guidelines for the treatment of AAP; therefore, further accumulation of EVT cases can provide evidence for its treatment plan and applicable cases.

Informed consent was obtained from the patient described in this case report, and the identity of the patient has been protected. Ethical approval was obtained (September 2, 2020; number: 20,202-30,902).

\section{References}

1. Ingoglia M, Pani S, Britton L, Hanakova M, Raja A, El-Mohtar K, et al. Ascending aortic pseudoaneurysm. J Cardiothorac Vasc Anesth. 2011;25:1098-100.

2. Sullivan KL, Steiner RM, Smullens SN, Griska L, Meister SG. Pseudoaneurysm of the ascending aorta following cardiac surgery. Chest. 1988;93:138-43.

3. Ghoreishi M, Shah A, Jeudy J, Pasrija C, Lebowitz J, Kaczorowski D, et al. Endovascular repair of ascending aortic disease in high-risk patients yields favorable outcome. Ann Thorac Surg. 2020;109:678-85.

4. De Boo DW, Mott N, Kavnoudias H, Walton A, Lyon SM. Endovascular closure of ascending aortic pseudoaneurysm with a type II Amplatzer vascular plug. Vasc Endovascular Surg. 2014;48:329-32.

5. Shammas NW, Chammas MZ, Robken J, Geiss D. Percutaneous closure of the aorto-ostial origin of a coronary artery saphenous bypass graft with a large pseudoaneurysm using the AMPLATZER muscular ventricular septal defect occluder. Int J Angiol. 2017;26:196-200. 